\title{
Genesis
}

Manuscrits - Recherche - Invention

$50 \mid 2020$

Aragon

\section{Quelques notes sur l'histoire du « Fonds Aragon »}

\section{Marie Odile Germain}

\section{OpenEdition}

\section{Journals}

Édition électronique

URL : https://journals.openedition.org/genesis/5327

DOI : 10.4000/genesis.5327

ISSN : 2268-1590

\section{Éditeur :}

Presses universitaires de Paris Sorbonne (PUPS), Société internationale de génétique artistique littéraire et scientifique (SIGALES)

\section{Édition imprimée}

Date de publication : 15 juillet 2020

Pagination : 125-127

ISBN : 979-10-231-0679-4

ISSN : 1167-5101

\section{Référence électronique}

Marie Odile Germain, « Quelques notes sur l'histoire du « Fonds Aragon » », Genesis [En ligne], 50 |

2020, mis en ligne le 15 juillet 2021, consulté le 03 septembre 2021. URL : http://

journals.openedition.org/genesis/5327 ; DOI : https://doi.org/10.4000/genesis.5327 


\title{
Quelques notes sur l'histoire du "fonds Aragon»
}

\author{
Marie Odile Germain
}

$\mathrm{P}$ armi les grands corpus littéraires conservés à la Bibliothèque nationale de France, le fonds Elsa Triolet - Louis Aragon occupe une place singulière, tant pour le département des Manuscrits que pour l'ITEM... Ne serait-ce d'abord qu'en raison de son statut juridique : confié par Louis Aragon au CNRS en 1976, il ne fut déposé par le CNRS à la BnF qu'une trentaine d'années plus tard, la convention de dépôt entre les deux établissements étant signée en 2006. Si de nombreuses œuvres d'Aragon figurent dans les collections de la BnF (dont certaines d'importance particulière, comme les manuscrits des Beaux Quartiers, d'Aurélien, d'Henri Matisse, roman, ou les textes automatiques confiés à André Breton, et parfois d'acquisition récente, comme le Traité du style), si d'autres richesses sont conservées à la Bibliothèque littéraire Jacques Doucet («trésor» dont Aragon rappelait qu'il fut «le rassembleur initial»), la donation et le legs du fonds Triolet-Aragon (FTA) représentent à eux seuls un apport immense. Le volume en est considérable : 60000 feuillets (notes, manuscrits, dactylographies, épreuves), matériaux de choix pour les amateurs de genèse textuelle, même si tous les états n'y figurent pas et s'il est parfois difficile chez Aragon de distinguer un premier jet d'un début de mise au net tant sont grandes sa rapidité et sa virtuosité d'écriture. L'intérêt en est majeur, par les œuvres conservées (du premier écrit de l'enfance jusqu'à l'ultime Théâtre/Roman, en passant par La Semaine sainte, La Mise à mort, Blanche ou l'oubli, pour ce qui concerne les romans, et pour la poésie de Feu de joie ou du Paysan de Paris au Fou d'Elsa, en passant par Le Crève-cour et Le Roman inachevé). D'intérêt majeur aussi la correspondance reçue, soit environ 10000 lettres aux signatures illustres (Breton, Gide et Matisse parmi tant d'autres), les archives personnelles, la documentation historique, ou politique (celle, entre autres, du Comité national des écrivains), etc. Et la provenance est évidemment prestigieuse, puisque ces archives ont été formées par leur propre auteur.

L'histoire, en ses débuts du moins, est connue de tous les «aragoniens »; ses principaux protagonistes l'ont évoquée chacun à sa manière : Jean Ristat, qui fut le secrétaire de l'écrivain et devint son héritier et légataire universel, en même temps que le premier chercheur affecté au classement de ses archives; Michel Apel-Muller, spécialiste d'Elsa Triolet, proche lui aussi d'Aragon, directeur du fonds Triolet-Aragon, qui l'a rappelé dans le long entretien accordé à Luc Vigier et Maryse Vassevière, et accessible sur le site en ligne de l'ERITA ${ }^{1}$; Renate Lance, chercheur détachée à l'ITEM, qui en a fourni un exposé détaillé, en introduction au précieux catalogue du fonds qu'elle mena à bien au cours des années 1980-1990². Quant à Louis Aragon, il lui a donné sa forme mythique dans la fameuse allocution prononcée lors de la remise de ses manuscrits au CNRS en mai 1977. L'allocution sera reprise en 1979, en ouverture des Essais de critique génétique, ce premier manifeste collectif des spécialistes de genèse des textes, comme dit le programme du colloque; et son titre est flamboyant : «D'un grand art nouveau : la recherche ${ }^{3}$ ». La recherche élevée au rang des beaux-arts, et le fonds Aragon en drapeau du mouvement de la génétique des textes! C'est dire si le don et le legs du fonds Aragon sont étroitement liés à l'histoire de l'ITEM et de son ancêtre le CAM (Centre d'analyse des manuscrits).

Tout s'était noué en juin 1976, quand l'écrivain manifesta solennellement et par écrit sa décision de «remettre à la nation française, quelle que soit la forme de son gouvernement, le legs littéraire d'Elsa Triolet et l'ensemble des manuscrits et documents en sa possession qui appartiendront au Centre national de la recherche scientifique», et d'en «confier la conservation et l'exploitation scientifique au

1. Maryse Vassevière et Luc Vigier, «Entretien avec Michel Apel-Muller», Recherches croisées Aragon / Elsa Triolet, $\mathrm{n}^{\circ}$ 9, Presses universitaires de Strasbourg, 2004, p. 107-147 (en ligne sur www.louisaragon-elsatriolet.org).

2. Renate Lance-Otterbein, Le Fonds Elsa Triolet-Aragon du CNRS, un legs à la nation française, Catalogue, t. I, 336 p. Un exemplaire dactylographié est consultable au département des Manuscrits de la BnF.

3. Louis Aragon, «D'un grand art nouveau : la recherche», Essais de critique génétique, Paris, Flammarion, 1979, p. 7-19. 
Centre d'histoire et d'analyse des manuscrits modernes ». Comment ne pas noter l'incroyable - et fière - ressemblance avec le geste, et la geste, inaugural(e), de Victor Hugo un siècle plus tôt : dans le célèbre codicille testamentaire qui instaurait la Bibliothèque nationale de Paris, «qui sera un jour la bibliothèque des États-Unis d'Europe», légataire de «ses manuscrits et de tout ce qui sera trouvé écrit et dessiné par lui»? Mais le choix par Aragon d'un autre destinataire, le CNRS, renouvelait la proposition : il traduisait l'attention de l'écrivain à son propre travail d'élaboration textuelle, le double souci de maitrise et d'ouverture de ses archives - allant de pair avec le soin qu'il avait pris à rééditer de son vivant ses œuvres posthumes, à grand renfort de préfaces, commentaires et remises à jour - et impliquait plus directement le monde de la recherche à travers les récents développements de la critique génétique.

Dans son entretien à bâtons rompus, Michel ApelMuller raconte comment une telle décision s'est imposée à l'écrivain, d'abord de façon négative :

Pour ses papiers il ne voulait pas entendre parler de la Bibliothèque nationale du fait de la durée trop longue entre le dépôt et la consultation, souvent un demi-siècle. Il voulait moins encore entendre parler du fonds Doucet, mais pour des raisons qui tenaient à son passé, il n'avait pas de bons souvenirs du fonds Doucet...

La décision fut aussi comme un précipité de hasard et de nécessité : les attentes d'Aragon, les conseils de ses proches, au premier rang desquels Jean Ristat et Michel Apel-Muller, et le déclic, ou le kairos, provoqué par la rencontre d'un professeur d'université, Jean Peytard, et de Louis Hay, fondateur du CAM, lors d'une soutenance de thèse... Ils évoquent le sort des manuscrits d'Aragon. Le CNRS dira très vite son intérêt, et Aragon approché donnera son accord de principe.

«Il n'a pas été besoin de longues conversations pour me convaincre, rappelle l'écrivain dans son discours de mai 1977 à l'adresse du Directeur du CNRS. Votre collaborateur pourrait vous le dire. J'ai été plus tôt convaincu qu'il ne l'attendait. » Car, avait-il reconnu, «par extraordinaire, rien ne pouvait être plus voisin de mes désirs. Cela impliquait naturellement la nécessité de pouvoir mettre sous les yeux des lecteurs passionnés que sont les vrais chercheurs, non pas le texte en état de publication, toute retouche faite, et mené par son auteur à l'état de livre, c'est-à-dire de marchandises livrées, mais, surprises pendant son écriture, les hésitations de l'auteur, ses secrètes démarches, ses erreurs mêmes... voire ses brouillards, encore un air de brouillon, par-ci, par-là...»

Ce jour-là, la remise des manuscrits fut pourtant plus formelle que réelle... Évoquant le sens de sa démarche, Aragon y disait à la fois l'effort que lui demandait cette donation et l'espoir qu'il y mettait :

J'apporte peu à peu, imaginez-vous, le temps de rechercher dans mes armoires, ma bibliothèque, mes tiroirs, la diaspora de mes anciennes pensées, la dispersion de mes écrits, ce qu'il en reste au moins, et c'est comme un monde à tirer de l'enfer..., pour mettre à la disposition des chercheurs d'aujourd'hui, non pas seulement un certain nombre de manuscrits, au sens des éditeurs, mais les papiers de ma vie mentale, les dossiers complexes sans lesquels, à mon sens, ce qu' on appelle le manuscrit n'est que bien peu de choses...

L'entrée de la totalité des papiers d'Aragon ne sera effective que près de dix ans plus tard, en 1986, après le décès de l'écrivain en 1982. Et c'est à Jean Ristat, l'héritier, que reviendra la constitution du fonds - un fonds qui fut abrité dans des locaux que la $\mathrm{BnF}$ avait mis à la disposition des chercheurs chargés de les traiter, à l'emplacement même de la maison où Stendhal écrivit Le Rouge et le Noir : coöncidence providentielle qu'Aragon n'avait pas manqué de noter. C'est là que les «aragoniens », et d'abord Jean Ristat, Michel Apel-Muller, puis Renate Lance et combien d'autres, ont travaillé à rassembler, classer et cataloguer l'ensemble du fonds.

Lorsque, pour des raisons immobilières et administratives, il fallut trouver un lieu d'accueil plus pérenne au début des années 2000, l'ITEM choisit de transférer ce précieux héritage auprès des collections du département des Manuscrits : il n'y eut qu'à traverser la rue de Richelieu. Le déménagement ne posa guère de problème, grâce à l'efficacité de Danièle Maïsetti de l'ITEM, et à sa connaissance du fonds, que tous les chercheurs avaient appréciée. Et la convention de dépôt, qui laissait au CNRS la propriété du fonds, donna à la $\mathrm{BnF}$ la charge de la conservation et de la communication de tous les documents Triolet-Aragon, à l'exception de l'iconographie qui rejoignit la bibliothèque des deux écrivains conservée au Moulin de Saint-Arnoult-en-Yvelines : je pris donc le relais de nos collègues de l'ITEM dans un rôle plus 
logistique qu'intellectuel, puisque le fonds lui-même avait été presque totalement classé et catalogué par ses précédents responsables, et en tout premier lieu par Renate Lance.

Un classement et un catalogage qu'elle avait marqués d'une ambition «génétique» : si les documents sont traditionnellement répartis par genre, et à l'intérieur par ordre chronologique des œuvres, les unités de description correspondent très exactement aux unités de conservation, et sont fondées sur des observations génétiques. L'informatisation n'en étant qu'à ses débuts au moment de ce long travail, l'inventaire se présente sous la forme d'une dactylographie sur papier, utilisant un traitement de texte non structuré, et le fichier des correspondances est composé de fiches bristol. En 2005, après le dépôt à la BnF, et sous l'impulsion de la Direction du livre, en accord avec l'ITEM, il fut décidé d'intégrer cet inventaire des manuscrits et les fichiers de correspondances dans le «Répertoire des manuscrits littéraires français du $\mathrm{XX}^{\mathrm{e}}$ siècle»: les notices saisies selon le format Opaline par une équipe de vacataires travaillant à la $\mathrm{BnF}$ devenaient accessibles dans la base PALME du Catalogue collectif de France (CCFR) : elles le sont toujours, mais ce qui était une forme de modernité il y a une douzaine d'années, devrait sans doute être repensé avec un système d'encodage semblable à celui de la base «archives et manuscrits », plus souple et plus performant ! Cela n'empêche pas les lecteurs de consulter ce fonds, qu'ils se livrent aux délices de la génétique, de l'histoire intellectuelle ou à des travaux éditoriaux, à commencer par ceux de la Pléiade dont les volumes, modèles d'acribie critique, regorgent de notes tirées du fameux «FTA».

Cette présentation n' aura fait que survoler l'histoire du fonds et les différents avatars de son aventureuse existence. Car l'histoire des grands fonds est toujours une aventure. Et nous voilà arrivés à l'année 2008, date historique aussi s'il en est, puisqu'il s'agit de la date de naissance de la nouvelle équipe Aragon de l'ITEM...

(Texte écrit pour le Cinquantenaire de l'ITEM et prononcé à cette occasion, le 20 octobre 2018)

MARIE Odile GeRMAIN, conservateur général honoraire au département des Manuscrits de la BnF, a été chargée de nombreux fonds littéraires contemporains, dont le fonds Aragon. Elle a publié Les plus belles lettres manuscrites de la langue française (1992), Trésors de la Bibliothèque nationale de France, II (2000), Genèses Généalogies Genres : autour de l'œuvre d'Hélène Cixous (2006), et organisé plusieurs expositions : Brouillons d'écrivains (2001), Michel Butor, l'écriture nomade (2006), Antonio Tabucchi ou le fil de l'écriture (2014), Les Écritures de Roland Barthes (2015). 